\title{
Relationship of lisinopril with kallikrein-kinin system in hypertensive patients in Erbil city, I raq
}

Abstract

Background and objective: Hypertension is characterized by a persistent, progressive elevation in blood pressure. Oxidative stress and endothelial dysfunction have a role in the pathogenesis of hypertension through interaction with the elements of the renin-angiotensin system. This study aimed to examine the effects of lisinopril on mean arterial pressure and biosubstances of Kallikrein-kinin-system (bradykinin), endothelial dysfunction (nitric oxide), and oxidative stress (malondialdehyde).

Methods: A clinical trial was conducted in Erbil city from December $1^{\text {st }}, 2015$ to August $10^{\text {th }}, 2016$. The study included 65 patients with essential hypertension and 25 apparently healthy subjects; their ages were in between 18 and 55 years. The patients were receiving $10 \mathrm{mg}$ lisinopril orally per day for six weeks as a starting dose.

Results: At hypertension diagnosis, patients were with lower bradykinin and nitric oxide levels when compared with apparently healthy subjects; however, malondialdehyde level showed no significant difference when compared with of healthy subjects. After six weeks patients treatment, comparing bradykinin, nitric oxide, and malondialdehyde mean levels with their baselines, showed that significantly increased in bradykinin and nitric oxide $(P<0.01)$ and significantly decreased in malondialdehyde $(P<0.01)$. On the other hand, the differences between after treatment and healthy subjects had no significant difference, except bradykinin. Eventually, during treatment, the mean arterial pressure was significantly lowered.

Conclusion: in addition to the significant lowering of blood pressure, lisinopril $10 \mathrm{mg}$ daily for six weeks can significantly elevate kallikrein-kinin system and endothelial dysfunction markers, and significantly lowered in oxidative stress marker in hypertensive Kurd patients in Erbil city.

Keywords: Lisinopril; Hypertension; Bradykinin; Nitric oxide; Malondialdehyde.

\section{Introduction}

Hypertension (HTN) is characterized by a persistent progressive elevation in blood pressure. HTN is commonly defined as systolic blood pressure (SBP) $\geq 140 \mathrm{mmHg}$ and diastolic blood pressure (DBP) $\geq 90$ $\mathrm{mmHg} .{ }^{1}$ It is typically asymptomatic state, with time it would disturb the renal, cardio- and neuro-vascular system. HTN is etiologically classified into essential hypertension (EH) and secondary hypertension. $\mathrm{EH}$ is more common than secondary that affects 90-95 percent of persons who have HTN, resulting from the interaction between genetic and environmental factors. Secondary hypertension consists 5-10 percent of hypertensive patients with detectable and treatable causes that may results from deterioration in the hormonereliant endocrine system. Majority of trials emphasize on endothelial dysfunction (ED) in patients with $\mathrm{EH}$ as a result of reduction in nitric oxide (NO) synthesis owing to oxidative stress (OS). Impairment of NO and overproduction of angiotensin II (Ang II) will produce atherosclerosis through precipitating lipid and creation of fibrolipid

* Directorate of Health, Erbil, I raq.

** Department of Pharmacology and Toxicology, College of Pharmacy, Hawler Medical University, Erbil, I raq. 
plaques in the vessels. ${ }^{2}$ Nervous mechanisms, sympathicoadrenal system, vasopressin, and renin- angiotensinaldosterone system (RAAS) are the general agents that control vascular tonicity. ${ }^{3}$ RAAS plays a main role in the pathogenesis of hypertension, ${ }^{4}$ through a series of changes by the action of angiotensin converting enzyme (ACE) to convert angiotensin I (Ang I) into Ang II, ${ }^{5}$ which is a main character in coordinating the cardiovascular physiology. ${ }^{6}$ Also OS has a role in the pathogenesis of HTN through interaction with the elements of the RAS; upregulating renal Ang I receptor expression, thereby sodium retention and elevation of BP. ${ }^{7}$ The kallikrein-kinin system (KKS) by the action of kallikrein can produce kinins from kininogen. KKS, for e.g. bradykinin (BK), has a practical role in the arrangement of blood pressure by triggering particular body receptors to exert its pharmacological action. By the action of ACE, RAAS and KKS participate in the cardiovascular homeostasis to produce Ang II and breakdown BK; these are contributed in the arrangement of mean arterial pressure (MAP). ${ }^{8}$ Virdis et al. indicated that ACE inhibitors can importantly restore endothelial function, increment of NO bioavailability, as well as significant lowering in plasma markers of OS, specifically malondialdehyde (MDA). ${ }^{9}$ Also showed that lisinopril increased vasodilation by the action of $\mathrm{BK}$ in patients with $\mathrm{EH}$. Previous studies have demonistrated that high blood pressure has a link with KKS, ED, and OS. This study aimed to determine the effect of lisinopril on MAP and to examine the relationship of lisinopril with parameters of $\mathrm{BK}, \mathrm{NO}$, and MDA in hypertensive patients.

\section{Methods}

Sixty five patients suffering from $\mathrm{EH}$ were enrolled in this study in Rizgary Teaching Hospital at the capital of Iraqi Kurdistan region, Erbil. Their ages were between 18 and 55 years with mean ages and SEM

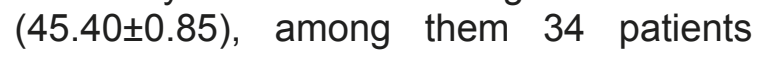

$(52.3 \%)$ were females. These patients received $10 \mathrm{mg}$ lisinopril orally per day for 6 weeks as starting dose. Along which, 25 apparently healthy subjects participated in the study as control. Their mean ages and SEM were $(38.4 \pm 2.03)$, and 13 subjects $(52 \%)$ of them were females. Hypertensive patients with SBP $\geq 160 \mathrm{mmHg}$ and $\mathrm{DBP} \geq$ $90 \mathrm{mmHg}$ were included in the study according to recent report from the panel members appointed to the Eighth Joint National Committee (JNC8). ${ }^{10}$ Informed consent was obtained from all participants before being interviewed and filled the questionnaires. The study was approved by the Research Ethics Committee at the College of Pharmacy, Hawler Medical University. For patients and healthy subjects, six parameters were addressed, three of six parameters regarded as BK, $\mathrm{NO}$, and MDA. Other parameters include SBP, DBP, and MAP. $5 \mathrm{ml}$ of venous blood were drawn by venipuncture with syringe and tourniquet from each participant after overnight fasting and transferred the blood sample to an anticoagulant free tube and left for forty minutes to clot, then they were centrifuged for 5 minutes at 3000 round per minute (Hettich, Rotofix 32, Germany). After the separation of serums they were stored in eppendorf tube at $\left(-20^{\circ} \mathrm{C}\right)$ until the time of analysis. From which $\mathrm{BK}, \mathrm{NO}$, and MDA parameters were used (HANGZHOU EASTBIPHARM CO. LTD, USA, Assembled by china). This assay employs the quantitative sandwich enzyme immunoassay technique (Anthos/biochrom Ltd, Cambridge, England). Antibody for each of BK, NO, and MDA seperatedly, has been precoated onto a microplate. Standards and samples are pippeted into the wells with a Horseradish Peroxidase (HRP) conjugated antibody specific for them. Following a wash to remove any unbound reagent, a substrate solusion is added to the walls and color develops in proportion to the amount of each of BK, $\mathrm{NO}$, and MDA in separatedly, bound in the initial step. The color development is stopped and the intensity of the color is 
measured using a microplate reader (Anthos 2010 - biochrom/England). Data are presented as mean \pm standard error of mean (SEM). Statistical evaluation was performed using paired t-test to compare the means before and after treatments among one group patients, and using independent $t$-test to compare the means of healthy and patient groups. Results were considered significant when $P$ value $<0.01$. The data were processed with SPSS statistics (statistical package for the social science software) version 22.

\section{Results}

Table 1 showed that SBP, DBP, and MAP levels were significantly higher when compared with healthy subjects group. Additionally, comparing SBP, DBP, and MAP mean levels before and after treatment in patients, showed that there were significant changes $(P<0.01)$. Finally, the differences between after treatments and control groups have also significant difference. On the other hand the comparison of baseline and after treatment measurements of BK, NO, and MDA levels (Table 2) in hypertensive patients were done and comparing such parameter mean levels with of healthy subjects. Data also demonstrated that mean serum BK and NO levels were lower when compared with healthy subjects group. In contrast, the mean serum MDA level in hypertensive group showed no significant difference when compared with healthy subjects group. Moreover, comparing BK, NO, and MDA mean levels before and after treatment in patients, showed that there were significant changes $(P<0.01)$. Eventually, the differences between after treatments and control groups have no significant difference, except BK.

Table 1: Baseline and after treatment measurements of SBP, DBP, and MAP levels in hypertensive patients compared to healthy subjects.

\begin{tabular}{lccc}
\hline Parameters & \multicolumn{2}{c}{$\begin{array}{c}\text { Lisinopril group }(\mathbf{n}=65) \\
\text { After } \mathbf{6} \text { weeks } \dagger\end{array}$} & $\begin{array}{c}\text { Apparent healthy } \\
\text { group }(\mathbf{n}=\mathbf{2 5})^{*}\end{array}$ \\
\hline SBP $(\mathrm{mmHg})$ & $166.5 \pm 0.72^{\prime \prime}$ & $137 \pm 1.14 "$ & $125 \pm 1.4 "$ \\
DBP $(\mathrm{mmHg})$ & $98.49 \pm 0.7^{\prime \prime}$ & $86.1 \pm 0.7^{\prime \prime}$ & $75.2 \pm 1.48 "$ \\
MAP $(\mathrm{mmHg})$ & $121.15 \pm 0.6^{\prime \prime}$ & $103 \pm 0.7^{\prime \prime}$ & $85.6 \pm 2.42^{\prime \prime}$ \\
\hline
\end{tabular}

† Paired-samples $t$ test; between baseline and after treatment.

¥ Independent-samples t test; between baseline and healthy.

* Independent-samples t test; between after treatment and healthy.

" $P<0.001$.

Table 2: Baseline and after treatment measurements of $\mathrm{BK}, \mathrm{NO}$, and MDA levels in hypertensive patients compared to healthy subjects

\begin{tabular}{|c|c|c|c|}
\hline \multirow{2}{*}{ Parameters } & \multicolumn{2}{|c|}{ Lisinopril group $(n=65)$} & \multirow{2}{*}{$\begin{array}{l}\text { Apparent healthy } \\
\text { group }(n=25)^{\star}\end{array}$} \\
\hline & Baseline $\ddagger$ & After 6 weeks $†$ & \\
\hline $\mathrm{BK}$ (ng/ml) & $3.04 \pm 0.68 "$ & $9.9 \pm 2.01 "$ & $23 \pm 3.36 "$ \\
\hline $\mathrm{NO}(\mu \mathrm{mol} / \mathrm{L})$ & $36.25 \pm 5.74^{n}$ & $49 \pm 8.25^{\circ}$ & $91.54 \pm 34.61^{n}$ \\
\hline MDA $(\mathrm{nmol} / \mathrm{ml})$ & 8.94ะ2.83" & $5.4 \pm 1.85^{\prime \prime}$ & $4.56 \pm 1.07 n$ \\
\hline \multicolumn{4}{|c|}{$\begin{array}{l}\text { † Paired-samples t test; between baseline and after treatment. } \\
\text { † Independent-samples t test; between baseline and healthy. } \\
\text { * Independent-samples t test; between after treatment and healthy. } \\
\text { " } P<0.001 \text {. } \\
{ }^{o} P=0.014 \text {. } \\
\text { n non significant. }\end{array}$} \\
\hline
\end{tabular}




\section{Discussion}

Hypertension is one of the most common diseases worldwide and a major modifiable risk factor for cardiovascular disease, its treatment and control rates are still suboptimal. ${ }^{11}$ In current study, it was revealed that hypertensive patients at diagnosis had significantly higher levels of SBP, DBP, and MAP compared with those healthy subjects (Table 1). Lisinopril is one of the most commonly prescribed antihypertensive agent is effective as monotherapy. ${ }^{12}$ Our results showed that lisinopril is substantially reduced the SBP, DBP, and MAP of patients during six weeks. A recent Kurdish study supports our data was done in Erbil city by Muslih, fulfilled with a significant decrement in MAP level during a week in hypertensive patients. ${ }^{13}$ Similarly, Saul et al. study showed that HTN had direct correlation with $\mathrm{BK}, \mathrm{NO}$, and MDA; lowers BK and NO, and elevates MDA, ${ }^{14}$ that agreed with the present study. The accumulating evidences suggest that potentiating the activity of KKS is made by suppressing presumably via production of $\mathrm{NO}$ as well as reduction of OS. ${ }^{15}$ Furthermore, Schulz et al. concluded that OS and ED are consistently observed in hypertensive subjects. ${ }^{16}$ Lisinopril treatment as an ACEI used for lowering blood pressure; manipulates with KKS as its adverse effect through which regulates endothelial $\mathrm{NO}$ and can reduces $\mathrm{OS}^{17}$ This evidence supports us in conducting hypertensive patients to receive lisinopril for the purpose of substantial elevating in $\mathrm{BK}$ and NO, and lowering in MDA levels $(P<0.001)$. Inconsistent with our study, Kohno et al. showed that BK is lowered but not statistically significant. ${ }^{18}$ Kaminskii et al. found that hypertensive patients received lisinopril were associated with significant increase in nitrate level. ${ }^{19}$ Similarly, Kosenko et al. investigated the effect of lisinopril on nitrate level among 29 hypertensive patients in Finland who visited health centers. Of note, $\mathrm{NO}$ is a highly unstable free radical and is oxidized rapidly to nitrate in circulating blood. ${ }^{20}$ With regards to the correlation between lisinopril treatment and MDA level, the current study revealed that MDA in hypertensive patients is lowered by the effect of lisinopril treatment. There was a significant decrement in its value; however, it observed high when compared with the MDA level in apparent healthy subjects. Some studies support our data. For instance, Velayutham et al. examined the relationship of lisinopril treatment with MDA parameter. ${ }^{21}$ They reported that MDA level was significantly lowered in hypertensive patients with supratentorial brain tumors after receiving half strength of lisinopril (5 mg). Lisinopril, along with its blood lowering effects can influence on the other actions. For instance; BK as KKS has been enhanced in any patient who treated with lisinopril drug as well as can successfuly increases NO; moreover OS is also improved. Thus, augmentation in each of $\mathrm{BK}$ and $\mathrm{NO}$ magnitudes in addition to diminution in OS marker levels can productively improve high blood pressure. ${ }^{17}$ Multiple studies have shown that ACE inhibitors improve endothelial function, probably by increasing $\mathrm{BK}$ in the arterial wall and decreasing $\mathrm{OS}^{22}$ Moreover, Kirbas demonstrated that lisinopril effects on NO and MDA levels were not observed in L-Name $\left(\mathrm{N}_{3}\right.$-nitroL-Arginiine Methyl Ester hydrochloride) induced hypertensive rats. ${ }^{23}$ One of the reasons behind the discrepancy of data among these studies is genetic variation, which means that all of the studies have not been conducted in the same country or population or area. Besides, subjects in this study distinctly were hypertensive patients. Furthermore, potassium plays an important role in the lowering blood pressure that may be elevated with the lisinopril treatment. We propose that potassium may further lowers blood pressure, this notion is supported by evidence of Albarwani and his collegues; ${ }^{24}$ found that potassium can reduces blood pressure. 


\section{Conclusion}

The present study concludes that lisinopril significantly reduces the blood pressure and malondialdehyde as well as significantly increases the bradykinin and nitric oxide in Kurd hypertensive patients in Erbil city.

\section{Competing interests}

The authors declare that they have no competing interests.

\section{References}

1. Williams SF, Nicholas SB, Vaziri ND, Norris KC. African Americans, hypertension and the renin angiotensin system. World J Cardiol 2014; 6(9):878-89.

2. Dufton J. The pathophysiology and pharmaceutical treatment of hypertension. PharmCon, Inc; 2011.

3. Kasko M, Budaj M, Hulin I. Harmful or helpful hypertension-pathophysiological basis. Slovakia: Bratislava Comenius University. Genetics and Pathophysiology of Essential Hypertension. Khullar M, editors. Hypertension, 1st ed. Rijeka, Croatia: InTech; 2012. P. 9.

4. Moon JY. Recent update of renin-angiotensinaldosterone system in the pathogenesis of hypertension. Electrolyte Blood Press 2013; 11(2):41-5.

5. Unger T, Paulis L, Sica DA. Therapeutic perspectives in hypertension: novel means for renin-angiotensin-aldosterone system modulation and emerging device-based approaches. Eur Heart J 2011; 32(22):2739-47.

6. Flores-Munoz M, Work LM, Douglas K, Denby L, Dominiczak AF, Graham D, et al. Angiotensin(1-9) attenuates cardiac fibrosis in the stroke-prone spontaneously hypertensive rat via the angiotensin type 2 receptor. Hypertens 2012; 59(2):300-7.

7. Luo H, Wang X, Chen C, Wang J, Zou X, Li C, et al. Oxidative stress causes imbalance of renal renin angiotensin system (RAS) components and hypertension in obese Zucker rats. J Am Heart Assoc 2015; 4(2):e001559.

8. Camargo AC, lanzer D, Guerreiro JR, Serrano SM. Bradykinin-potentiating peptides: beyond captopril. Toxicon 2012; 59(4):516-23.

9. Virdis A, Ghiadoni L, Taddei S. Effects of antihypertensive treatment on endothelial function. Curr Hypertens Rep 2011; 13(4):27681.

10. Bell K, Twiggs J, Olin BR. Hypertension: The Silent Killer: Updated JNC-8 Guideline Recommendations. Alabama pharmacy association: Contining education; 2015. P. 1-8.

11. Al Ghatrif M, Kuo YF, Al Snih S, Raji MA, Ray LA, Markides KS. Trends in hypertension prevalence, awareness, treatment and control in older Mexican Americans, 1993-2005. Ann Epidemiol 2011; 21(1):15-25.

12. Gu Q, Burt VL, Dillon CF, Yoon S. Trends in antihypertensive medication use and blood pressure control among United States adults with hypertension the National Health and Nutrition Examination Survey, 2001 to 2010. Circ 2012; 126(17):2105-14.

13. Muslih Al. Reduction of mean arterial pressure and proteinuria by the effect of aceis (lisinopril) in Kurdish hypertensive patients in Hawler city. Glob J Health Sci 2012; 4(5):14.

14. Saul SM, Duprez DA, Zhong W, Grandits GA, Cohn JN. Effect of carvedilol, lisinopril and their combination on vascular and cardiac health in patients with borderline blood pressure: the DETECT Study. J Hum Hypertens 2013; 27(6):362-7.

15. Kayashima $\mathrm{Y}$, Smithies $\mathrm{O}$, Kakoki $\mathrm{M}$. Kinins-The Kallikrein-Kinin System and Oxidative Stress. Curr Opin Nephrol Hypertens 2012; 21(1):92-6.

16. Schulz E, Gori T, Münzel T. Oxidative stress and endothelial dysfunction in hypertension. Hypertens Res 2011; 34(6):665-73.

17. Regoli D, Gobeil F. Critical insights into the beneficial and protective actions of the kallikreinkinin system. Vascul Pharmacol 2015; 64:1-10.

18. Kohno M, Yokokawa K, Minami M, Yasunari K, Maeda $\mathrm{K}$, Kano $\mathrm{H}$, et al. Plasma levels of nitric oxide and related vasoactive factors following long-term treatment with angiotensin-converting enzyme inhibitor in patients with essential hypertension. Metab 1999; 48(10):1256-9.

19. Kaminskii YG, Suslikov AV, Tikhonova LA, Galimova MK, Ermakov GL, Tsvetkov VD, et al. Arginase, nitrates, and nitrites in the blood plas ma and erythrocytes in hypertension and after therapy with lisinopril and simvastatin. Biol Bull 2011; 38(5):446-52.

20. Kosenko E, Tikhonova L, Suslikov A, Kaminsky Y. Impacts of lisinopril and lisinopril plus simvastatin on erythrocyte and plasma arginase, nitrite, and nitrate in hypertensive patients. $\mathrm{J}$ Clin Pharmacol 2012; 52(1):102-9.

21. Velayutham PK, Adhikary SD, Babu SK, Vedantam R, Korula G, Ramachandran A. Oxidative stress-associated hypertension in surgically induced brain injury patients: Effects of $\beta$-blocker and angiotensin-converting enzyme inhibitor. J Surg Res 2013; 179(1):125-31.

22. Kelly AS, Gonzalez Campoy JM, Rudser KD, Katz H, Metzig AM, Thalin M, et al. Carvedilol $\square$ Lisinopril Combination Therapy and Endothelial Function in Obese Individuals With Hypertension. J Clin Hypertens 2012; 14(2):85-91.

23. Kirbas S. Effect of lisinopril on oxidative stress in brain tissues of rats with L-Name induced hypertension. Turk J Biochem 2013; 38(2):1638. 
Relationship of lisinopril with kallikrein-kinin .......

Zanco J. Med. Sci., Vol. 22, No. (2), August, 2018 https:/ / doi.org/ 10.15218/ zjms.2018.031

24. Albarwani S, Al-siyabi S, Al-husseini I, Al-ismail A, Al-lawati I, Al-bahrani I, et al. Lisinopril alters contribution of nitric oxide and $\mathrm{KCa}$ channels to vasodilatation in small mesenteric arteries of spontaneously hypertensive rats. Physiol Res 2015; 64:39-49. 